\title{
Successful surgical management of large conjunctival nevus in a 10-year-old child with resection and amniotic membrane transplantation
}

\author{
Tiong Kiew Ing, Shiivaa Manjare A/P Birapadian
}

Ophthalmology Department, Sarawak General Hospital, Kuching, Sarawak, Malaysia

\begin{abstract}
The purpose of this paper is to illustrate a case of large conjunctival nevus in a 10-yearold boy which was successfully treated with surgical excision and amniotic membrane transplant (AMT) reconstruction. The conjunctival nevus was initially noticed by the parents 1 year prior to presentation; they reported it had increased in size over the past 3 months. Slit-lamp examination revealed a pigmented conjunctival nevus measuring $5.5 \mathrm{~mm}$ vertically and $6.5 \mathrm{~mm}$ horizontally, with well-demarcated margins and presence of an intralesional cyst at the temporal bulbar conjunctiva, involving the limbus and encroaching onto the cornea. Complete resection of the conjunctival lesion and bulbar conjunctival reconstruction were performed. The histopathological examination showed conjunctival nevus. The wound healed well with vision of $6 / 6$ and no recurrence. Surgical resection combined with $A M T$ is a successful and an effective way to treat conjunctival nevus.
\end{abstract}

Keywords: amniotic membrane transplantation, conjunctival nevus, ocular surface reconstruction, surgical resection

\section{Introduction}

Conjunctival nevus is the commonest benign lesion of the ocular surface. It is usually found in the bulbar conjunctiva, caruncle, and plica semilunaris. Rarely, it is seen in the fornix, tarsus, or cornea. ${ }^{1}$ Amniotic membrane transplantation (AMT) was first performed in humans as a skin substitute to treat an open wound in 1910. ${ }^{2}$ AMT promotes epithelial wound healing, shows antibacterial, anti-inflammatory, antiscarring, and antiangiogenic properties.

Correspondence: Tiong Kiew Ing, MD, DR OPHTH, Ophthalmology Department, Sarawak General Hospital, Jalan Tun Ahmad Zaidi Adruce, 93586 Kuching, Sarawak, Malaysia.

E-mail: kiewing84@yahoo.com! 
To date, there are fewer than 20 publications of conjunctival nevus with AMT worldwide. We report a case of large conjunctival nevus in a child which required AMT following nevus resection.

\section{Case report}

A 10-year-old boy was referred to our department due to a pigmented conjunctival lesion in the left eye. The lesion was noticed by the parents 1 year prior to presentation; they reported it had increased in size over the past 3 months. There was no eye pain, redness, blurring of vision, change of color or shape of the conjunctival lesion. Examination revealed vision of $6 / 9$ in the left eye, with an interpalpebral conjunctival mass at the temporal region from 2 to 4 o'clock involving the limbus and encroaching onto the cornea (Fig. 1). It was a lightly pigmented mass, with well-demarcated margins measuring $5.5 \mathrm{~mm}$ vertically and $6.5 \mathrm{~mm}$ horizontally. There was presence of an intralesional cyst. The anterior chamber, ocular media, and fundus in the left eye were normal. Examination of the right eye was unremarkable.

Due to the recent increase in lesion size as well as cosmetic concerns, the patient and his parents requested surgery; hence, written informed consent for surgical excision and AMT was obtained. Complete surgical resection of the lesion with a tumor safety margin of $3.0 \mathrm{~mm}$ was performed using no-touch technique. The large conjunctival defect was reconstructed with single-layer amniotic membrane using the inlay technique, as shown in Fig 2. The amniotic membrane was secured

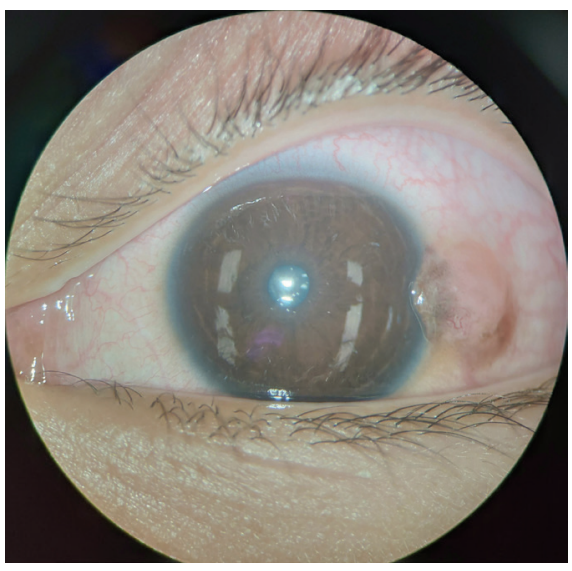

Fig. 1. Conjunctival nevus in the left eye measuring $5.5 \mathrm{~mm}$ vertically and $6.5 \mathrm{~mm}$ horizontally in the temporal region, involving the limbus and encroaching onto the cornea.

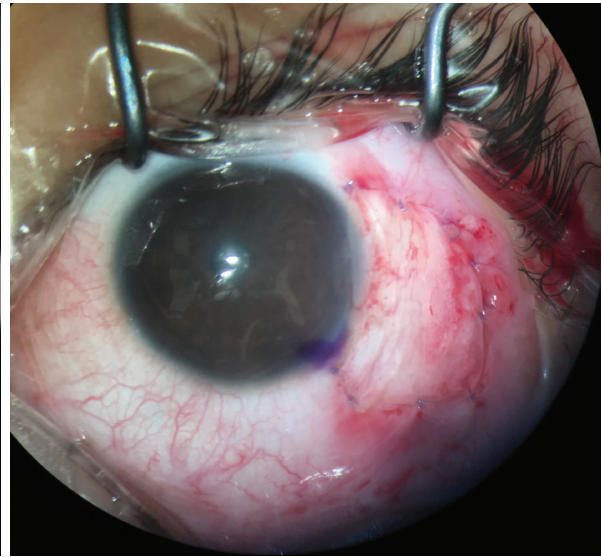

Fig. 2. AMT reconstruction was performed following excision of the conjunctival nevus. Graft was secured using interrupted 8-0 vicryl sutures. 


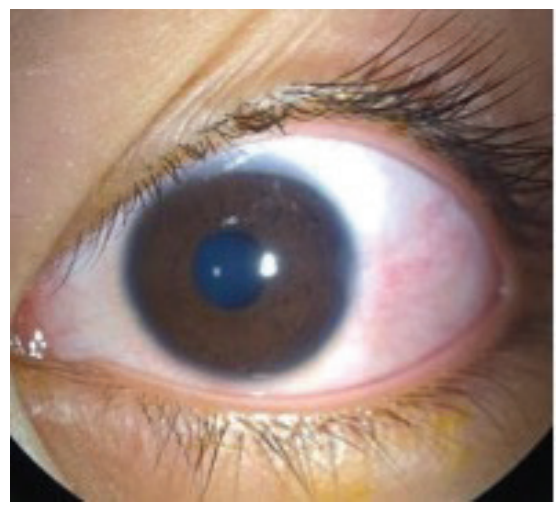

Fig. 3. Slit-lamp examination showed the surrounding host conjunctiva well integrated with graft tissue, with well-healed wound 4 months after surgery. There were no signs of recurrence or complications.

with 8-0 vicryl sutures and a bandage contact lens was applied. The histopathological examination showed conjunctival nevus. Postoperatively, the patient received topical corticosteroids ( $0.1 \%$ dexamethasone ophthalmic solution) and antibiotics ( $0.5 \%$ moxifloxacin hydrochloride ophthalmic solution) every 2 hours daily for a week, which was tapered to every 6 hours daily for the following week. Vision was recovered at $6 / 6$ with a well-healed wound and no recurrence of the lesion was noted (Fig. 3).

\section{Discussion}

Conjunctival nevus is the most common benign conjunctival lesion of the ocular surface among pediatric patients. It is most commonly seen in young white individuals, with mean age of patients being 32 years old. ${ }^{3}$ It is occasionally noticed at birth but more commonly it develops during childhood or adolescence, as in our patient. It displays a spectrum of variation from darkly pigmented to non-pigmented. Approximately $7 \%$ of patients may experience an increase in lesion size and approximately $5 \%$ may have a change in the nevus color. ${ }^{1}$ Rarely, it can be a precursor of malignant melanoma among the younger age group less than 18 years old. It is of utmost importance to monitor and look for any suspicious or warning signs of malignancy. Clinical characteristics of possible malignant transformation include color variation, margin irregularity, rapid growth, and changes in size, shape, and elevation. If the lesion encroaches onto the cornea, as was the case in our patient, it may lead to amblyopia in children or progress to malignancy. ${ }^{4}$

Local excision of the conjunctival lesion is recommended if there are suspicions 
of malignant changes, persistent ocular irritation, or cosmetic concerns; the latter was the case for our patient and his parents. The entire conjunctival lesion was removed via the no-touch technique with a tumor safety margin of $3.0 \mathrm{~mm}$. Complications may develop following resection of the conjunctival lesion, especially if there is a large conjunctival defect after excision. These include scar formation, symblepharon, granuloma, and partial or complete limbal stem cell deficiency. ${ }^{5}$ Hence, it is important to select an appropriate and reliable graft for ocular surface reconstruction in order to reduce the risk of these complications. The amniotic membrane is an excellent choice in restoring and reconstructing the ocular surface following large conjunctival lesion excision. The amniotic membrane consists of epithelium, thick basement membrane, and avascular stromal matrix. Its basement membrane exhibits many types of collagen; of these, type VII collagen is also found in conjunctival and corneal basement membranes, thus promoting cellular proliferation, migration, differentiation, and growth. ${ }^{6}$ On the other hand, the stromal matrix of the amniotic membrane contains hyaluronic acid and cytokines, which are essential for reduction of inflammation and inhibition of fibrosis. In other words, the amniotic membrane exerts potent anti-inflammatory, antiangiogenic, antifibrotic, and antimicrobial properties on the ocular surface. Therefore, it is increasingly being used to reconstruct the ocular surface for corneal or conjunctival defects and to promote healing of persistent epithelial defects, corneal ulcers, or ocular surface inflammation. In addition, it is rich with stem cells, thus promoting conjunctival and corneal epithelialization and migration of the stem cells without requiring any limbal stem cell transplant, especially in our case, where the limbus is involved. Rarely, pyogenic granuloma originating from sutures, hematoma beneath the graft, and hypopyon secondary to an immune reaction may be observed with AMT itself. ${ }^{7}$ However, no such complications were seen in our patient. A surgical excision combined with reconstruction via AMT is thus effective and economical for the treatment of large conjunctival lesions. ${ }^{8}$

In conclusion, early detection, proper diagnosis, and follow-up of conjunctival nevus are essential, especially in pediatric patients, to avoid amblyopia, persistent ocular irritation, and to detect any signs of malignant transformation. Urgent biopsy should be performed if there is any suspicion of malignancy. AMT is an excellent and reliable option for ocular surface reconstruction if there is a large conjunctival defect after excision of conjunctival lesion.

\section{Acknowledgements}

Informed consent was taken from the patient's parents for publication of this articles and accompanying photographs. 


\section{References}

1. Shields CL, Shields JA. Conjunctival Tumors in Children. Curr Opin Ophthalmol. 2007;18(5):351-360.

2. Davis JS. Skin transplantation. Johns Hopkins Hosp Rep. 1910;15:307-396.

3. Artur Zembowicz, Rajni V. Mandal, Pitipol Choopong. Melanocytic Lesions of the Conjunctiva. Arch Pathol Lab Med. 2010;134:1785-1792.

4. Khan L, Malukani M, Saxena A. Conjunctival Lesions: When Should We Perform Biopsy? Nepal J Ophthalmol. 2017;9(2):160-169.

5. Goktas SE, Katircioglu Y, Celik T, Ornek F. Surgical Amniotic Membrane Transplantation After Conjunctival and Limbal Tumor Excision. Arq Bras Oftalmol. 2017;80(4):242-246.

6. Malak TM, Bell SC. Differential expression of the integrin subunits in human fetal membranes. J Reprod Fertil. 1994;102(2):269-276.

7. Ahmad MS, Frank GS, Hink EM, Palestine AG, Gregory DG, McCourt EA. Amniotic Membrane Transplants in the Pediatric Population. J AAPOS. 2017;21(3):215-218.

8. Rock T, Bosmuller HC, Bartz-Schmidt KU, Rock D. Surgical Management of a Conjunctival Nevus with Amniotic Membrane Transplantation. Int Med Case Rep J. 2018;11:161-165. 